\title{
Globally Optimal Segmentation of Permanent-Magnet Systems
}

\author{
Insinga, Andrea Roberto; Bjørk, Rasmus; Smith, Anders; Bahl, Christian
}

Published in:

Physical Review Applied

Link to article, DOI:

10.1103/PhysRevApplied.5.064014

Publication date:

2016

Document Version

Publisher's PDF, also known as Version of record

Link back to DTU Orbit

Citation (APA):

Insinga, A. R., Bjørk, R., Smith, A., \& Bahl, C. (2016). Globally Optimal Segmentation of Permanent-Magnet Systems. Physical Review Applied, 5(6), [064014]. https://doi.org/10.1103/PhysRevApplied.5.064014

\section{General rights}

Copyright and moral rights for the publications made accessible in the public portal are retained by the authors and/or other copyright owners and it is a condition of accessing publications that users recognise and abide by the legal requirements associated with these rights.

- Users may download and print one copy of any publication from the public portal for the purpose of private study or research.

- You may not further distribute the material or use it for any profit-making activity or commercial gain

- You may freely distribute the URL identifying the publication in the public portal

If you believe that this document breaches copyright please contact us providing details, and we will remove access to the work immediately and investigate your claim. 


\title{
Globally Optimal Segmentation of Permanent-Magnet Systems
}

\author{
A. R. Insinga, R. Bjørk, A. Smith, and C. R. H. Bahl \\ Department of Energy Conversion and Storage, Technical University of Denmark-DTU, \\ Frederiksborgvej 399, DK-4000 Roskilde, Denmark
}

(Received 21 March 2016; revised manuscript received 26 May 2016; published 27 June 2016; publisher error corrected 1 July 2016)

\begin{abstract}
Permanent-magnet systems are widely used for generation of magnetic fields with specific properties. The reciprocity theorem, an energy-equivalence principle in magnetostatics, can be employed to calculate the optimal remanent flux density of the permanent-magnet system, given any objective functional that is linear in the magnetic field. This approach, however, yields a continuously varying remanent flux density, while in practical applications, magnetic assemblies are realized by combining uniformly magnetized segments. The problem of determining the optimal shape of each of these segments remains unsolved. We show that the problem of optimal segmentation of a two-dimensional permanent-magnet assembly with respect to a linear objective functional can be reduced to the problem of piecewise linear approximation of a plane curve by perimeter maximization. Once the problem has been cast into this form, the globally optimal solution can be easily computed employing dynamic programming.
\end{abstract}

DOI: 10.1103/PhysRevApplied.5.064014

\section{INTRODUCTION}

Permanent magnets play an important role in many scientific and industrial applications. Progress in the recent decades of research and development of new magnetic materials [1] has led to an immensely broadened range of applications of permanent magnets [2]. Applications of permanent magnets include rotary machines performing electromechanical energy conversion [3], magnetic actuators [4], magnetic gears [5], nuclear-magnetic-resonance devices [6,7], beam-focusing instruments [8,9], and wigglers and undulators [10].

In all these applications, it is always of the essence to optimize the design of the magnetic system, i.e., to increase the magnitude and precision of the generated field, and to reduce the volume and cost of the permanent-magnet materials. A wide range of different optimization techniques have been employed in this respect [11]. In general, it is advantageous to minimize the number of evaluations of the magnetic field solution, which is often performed with computationally expensive finite-element-method (FEM) techniques. However, because of the extent of the search space for this kind of problem, the advantage comes with a cost in terms of the optimality of the solution. Heuristic approaches, such as simulated annealing or, especially, genetic algorithms [12], are often preferred to deterministic algorithms in the attempt of avoiding to get stuck in local minima. In these schemes, there is always a trade-off between the computational complexity and the effectiveness of the algorithm in finding the optimal solution.

Other optimization approaches rely on the analytical calculation of the magnetic field $[6,9,13,14]$. In some cases,

\footnotetext{
*aroin@dtu.dk
}

it is possible to directly calculate the optimal solution based on analytical techniques. Even when this is not the case, the fast evaluation of the magnetic field solution allows the sampling of a large area of the considered search space, giving at least a close-to-optimal solution. The main limitation of this class of algorithms is the reduced dimension of the search space and the fact that only very few geometries are analytically solvable.

In this paper, we present an optimization method that can be applied to linear objective functionals and provides the globally optimal solution with single evaluation of the magnetic field solution and without the necessity of a starting guess. Restricting ourselves to linear objective functionals means that it is not possible to optimize, e.g., field homogeneity or, more generally, field quality. When the quality of the field with respect to a required distribution is critical, different strategies can be used to correct small field distortions $[8,15,16]$.

Our approach is based on the reciprocity theorem [17], an energy-equivalence principle of magnetostatics. The presented method does not share the limitations of the analytical techniques since it can be used in combination with FEM to calculate the magnetic field solution for geometries that are not analytically solvable. The result is always the optimal remanent flux density field over the specified design area. The use of the reciprocity theorem for magnet optimization has previously been limited to continuously varying remanences or presegmented structures $[18,19]$. In this paper, we show how the theorem can be used to calculate the globally optimal shape of each magnet piece of a segmented structure with a given number of pieces. This procedure allows for the development of a set of tools that greatly aid the design process of permanentmagnet systems. 
This methodology assumes that all the materials exhibit a linear magnetic behavior. For hard permanent magnets, this approximation is justified as long as the demagnetizing and transversal fields are not too intense with respect to the coercivity and the anisotropic field. The effect of the nonlinear demagnetization characteristics can be predicted with numerical approaches such as the ones described in Ref. [20]. However, the optimality of the configuration determined within the linear approximation is not guaranteed when these effects are taken into account.

\section{FRAMEWORK}

\section{A. Reciprocity theorem}

We begin by considering the reciprocity theorem, which considers two distinct magnetic systems labeled 1 and 2 [17]. The theorem states that the volume integral of the scalar product between the magnetization of system $1, \boldsymbol{M}_{1}$, with the field of system $2, \boldsymbol{H}_{2}$, is equal to the integral of the scalar product between the magnetization of system 2, $\boldsymbol{M}_{2}$, with the field of system $1, \boldsymbol{H}_{1}$,

$$
\int d^{3} x \boldsymbol{M}_{1}(\boldsymbol{x}) \cdot \boldsymbol{H}_{2}(\boldsymbol{x})=\int d^{3} x \boldsymbol{M}_{2}(\boldsymbol{x}) \cdot \boldsymbol{H}_{1}(\boldsymbol{x}) .
$$

The reciprocity theorem assumes that the fields are constant in time and vanish at infinity and that there are no electrical currents. The relation between magnetization $\boldsymbol{M}$, magnetic field $\boldsymbol{H}$, and flux density $\boldsymbol{B}$ is by definition $\boldsymbol{B}=\mu_{0}(\boldsymbol{H}+\boldsymbol{M})$.

We consider materials whose magnetic behavior is described by a linear constitutive relation $\boldsymbol{B}=\underline{\mu} \cdot \boldsymbol{H}+\boldsymbol{B}_{r}$, where $\underline{\underline{\mu}}$ denotes the permeability tensor, and $\boldsymbol{B}_{r}$ is the remanence vector. We also assume that the permeability tensor is symmetric and is the same in any point for systems 1 and 2 . When these conditions are fulfilled, the theorem can be expressed as

$$
\int d^{3} x \boldsymbol{B}_{r 1}(\boldsymbol{x}) \cdot \boldsymbol{H}_{2}(\boldsymbol{x})=\int d^{3} x \boldsymbol{B}_{r 2}(\boldsymbol{x}) \cdot \boldsymbol{H}_{1}(\boldsymbol{x}) .
$$

The reciprocity theorem can be used to solve magnet design problems by considering an empty air gap and by aligning the remanence everywhere with the field generated by a virtual magnet placed in this air gap. We consider an objective functional $\mathcal{S}$, which is linear with respect to $\boldsymbol{H}_{1}$, for example, maximizing the $x$ component of the field averaged over a certain region. In general, a linear functional can be expressed in integral form:

$$
\mathcal{S}\left[\boldsymbol{H}_{1}\right]=\int d^{3} x \boldsymbol{H}_{1}(\boldsymbol{x}) \cdot \boldsymbol{u}(\boldsymbol{x}),
$$

where $\boldsymbol{u}$ is an arbitrarily defined objective vector field. The integration domain can be reduced from the whole space to the region where the objective vector field $\boldsymbol{u}$ is nonzero.
This region will be denoted by $R_{g}$; it usually corresponds to an empty air gap. If the remanent flux density $\boldsymbol{B}_{r 2}$ of a virtual magnet is used as the objective vector field $\boldsymbol{u}$, Eq. (2) implies that $\mathcal{S}\left[\boldsymbol{H}_{1}\right]$ is also equal to

$$
\mathcal{S}\left[\boldsymbol{H}_{1}\right]=\int d^{3} x \boldsymbol{H}_{2}(\boldsymbol{x}) \cdot \boldsymbol{B}_{r 1}(\boldsymbol{x}),
$$

where $\boldsymbol{H}_{2}$ is the field generated by the virtual remanence $\boldsymbol{B}_{r 2}$, and $\boldsymbol{B}_{r 1}$ is the remanent flux density of the real magnet that is associated with the real field $\boldsymbol{H}_{1}$. The functional $\mathcal{S}$ has the dimensions of an energy. Again, the integration domain can be reduced to the region of space where the remanence of the real magnet is nonzero. This region denoted by $R_{m}$ and called the magnet design region is assumed not to overlap with $R_{g}$. Equation (2) applies since $R_{m}\left(R_{g}\right)$ will be the only region in which $\boldsymbol{B}_{r 1}\left(\boldsymbol{B}_{r 2}\right)$ is not zero. The use of the reciprocity theorem for the optimization of a magnetic system is schematically illustrated in Fig. 1.

The theorem provides the solution to the following problem: assuming that the norm of the remanent flux density $\boldsymbol{B}_{r 1}$ is fixed, determine its optimal direction in any point of the region $R_{m}$ such that the field $\boldsymbol{H}_{1}$ generated by $\boldsymbol{B}_{r 1}$ maximizes the integral of Eq. (3). The answer is evidently: the optimal remanence $\boldsymbol{B}_{r 1}$ should be aligned at every point with the virtual field $\boldsymbol{H}_{2}$ generated by $\boldsymbol{B}_{r 2}$. To illustrate this result, we consider a simple example in which we wish to maximize the field in a given direction inside a cylindrical region with infinite length. This region will then be occupied by a virtual magnet with uniform remanent flux density. If the direction of the virtual remanence is transversal to the axis of the cylinder, for example, in the positive $x$ direction, the field generated by it will lead to the

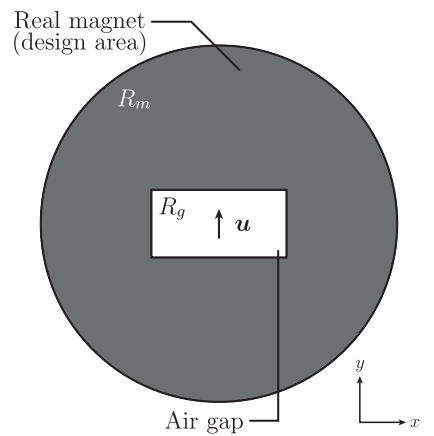

(a)

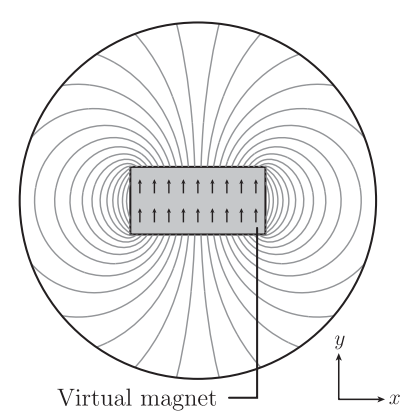

(b)
FIG. 1. (a) The use of the reciprocity theorem for magnet optimization is illustrated by considering a uniform objective field $\boldsymbol{u}$ oriented in the positive $y$ direction and defined over the rectangular air gap $R_{g}$. (b) The air gap $R_{g}$ is filled with a virtual magnet magnetized in the same direction of the objective vector field $\boldsymbol{u}$. The reciprocity theorem implies that the optimal remanence is aligned with the field generated by the virtual magnet in any point of the design area $R_{m}$. The field lines of the virtual field $\boldsymbol{H}_{2}$ are shown in (b). 
well-known Halbach cylinder solution [21], which, in cylindrical coordinates, is given by

$$
\boldsymbol{B}_{r 1}(r, \phi) \propto \cos (2 \phi) \hat{\boldsymbol{e}}_{x}+\sin (2 \phi) \hat{\boldsymbol{e}}_{y} .
$$

This case of the Halbach cylinder is considered in detail in Sec. IVA.

\section{B. Magnet border}

We now consider the external border of the magnet. An important observation regarding Eq. (4) is that the different pieces of magnet contribute independently to the value of the objective functional $\mathcal{S}$ : the optimal direction of the remanence in each point remains the same regardless of how the remanence is chosen elsewhere. The independence is a consequence of the fact that $\boldsymbol{H}_{1}$ is linear with respect to $\boldsymbol{B}_{r 1}$, and $\mathcal{S}$ is linear with respect to $\boldsymbol{B}_{r 1}$. Moreover, once $\boldsymbol{B}_{r 1}$ is aligned to $\boldsymbol{H}_{2}$ at a point $\boldsymbol{x}$, the integrand of Eq. (4) becomes $\left\|\boldsymbol{H}_{2}(\boldsymbol{x})\right\|\left\|\boldsymbol{B}_{r 1}(\boldsymbol{x})\right\| \geq 0$. This means that the contribution from that point of the magnet to the value of $\mathcal{S}$ is proportional to the norm of the virtual field, which can, thus, be interpreted as a weight factor of the corresponding site in the magnet design area [18]. This fact can be used to predict the optimal border between hard magnets and air.

As we explain in Sec. II A, in order for Eq. (2) to be true, the magnetic susceptibility $\chi_{m}$ must be the same for the real and the virtual system, implying that it is not possible to determine the optimal susceptibility field, i.e., the optimal distribution of materials. However, if the susceptibility of the permanent-magnet material is zero, as is approximately true, e.g., for rare-earth hard magnets, we are not forced to decide in advance which areas of the magnet design region are to be filled with magnet and which areas are to be filled with air. Instead, we can determine the optimal border between magnet and air using the amount of available permanent-magnet material as a constraint.

As the contribution to the value of $\mathcal{S}$ from a point of the design region is equal to $H_{2}=\left\|\boldsymbol{H}_{2}\right\|$, it is better to utilize the permanent-magnet material in areas where the norm of the virtual field $\boldsymbol{H}_{2}$ is larger. This implies that the optimal borders between hard magnet and air are given by the level surfaces of the scalar field $H_{2}(\boldsymbol{x})$.

For the case of the Halbach cylinder, the norm $\mathrm{H}_{2}$ of the virtual field $\boldsymbol{H}_{2}$ depends only on the radial coordinate and is proportional to $1 / r^{2}$. This implies that the optimal external border of the magnet is always a cylindrical surface which is coaxial with the inner cylindrical cavity.

In the examples that we present in Sec. IV, we assume that the susceptibility of the permanent-magnet material is zero. However, it should be stressed that this requirement is necessary only for the purpose of determining the optimal border between magnet and air. All the remaining optimality results presented in this work remain true for any tensor permeability field $\underline{\underline{\mu}}(\boldsymbol{x})$ which is symmetric.
Another interesting consequence of the reciprocity theorem concerns iron yokes, iron cores, or similar soft magnetic structures, which are often present in magnetic assemblies. If the magnet design region $R_{m}$ is adjacent to a region containing a material with very high permeability, e.g., iron, the virtual field $\boldsymbol{H}_{2}$ in $R_{m}$ will be almost normal to the border between the magnet and the highly permeable material (it will be exactly normal in the limit $\mu \rightarrow \infty$ ). Thus, if a permanent magnet is adjacent to an iron-filled region, it is never optimal for it to be magnetized in a direction that is not normal to the border between the magnet and iron, as this creates a short circuit for the flux lines to close without passing through the air-gap region. Therefore, the optimal remanence is always normal to the border between the magnet and iron.

\section{Energy considerations}

The method also suggests a practical technique for assembling systems composed of different pieces of permanent magnet. As it is explained in Ref. [22] for the case of the Halbach cylinder geometry, if a permanent magnet called the anchor is temporarily placed in the central air-gap region, it will exercise a force on the surrounding pieces of permanent magnet which will automatically align them in the Halbach configuration. The central anchor magnet plays the same role in this case as the virtual magnet in the optimization procedure presented here, and the value of $\mathcal{S}$ corresponds to the mutual energy between the anchor magnet and the surrounding segments. This fabrication method can be extended to many different cases besides the Halbach cylinder geometry, as long as the forces resulting from the mutual interaction between the different magnet pieces are negligible compared to the force due to the anchor magnet.

Furthermore, by allowing configurations where the norm of the remanence is not uniform, the algorithm can provide solutions that are also maximally efficient, in the sense that they maximize the fraction of magnetic energy that the permanent magnet is able to generate outside of itself [23]. More specifically, among all of the possible remanence distributions $\boldsymbol{B}_{r 1}$ in $R_{m}$ able to generate the same field $\boldsymbol{B}_{1}$ in $R_{g}$, the ideal remanence maximizes the figure of merit:

$$
\mathcal{M}=\frac{\int_{R_{g}} d^{3} x\left\|\boldsymbol{B}_{1}(\boldsymbol{x})\right\|^{2}}{\int_{R_{m}} d^{3} x\left\|\boldsymbol{B}_{r 1}(\boldsymbol{x})\right\|^{2}} .
$$

The sufficient conditions for maximal energy efficiency to occur are

(i) The magnet design region $R_{m}$ and the air-gap region $R_{g}$ are not overlapping.

(ii) The union of $R_{g}$ with $R_{m}$ is equal to the whole space, except for the regions enclosed by material with infinite permeability.

(iii) Inside $R_{g}$, the permeability is equal to $\mu_{0}$. 
(iv) Inside $R_{m}$, the permeability $\mu$ is uniform.

(v) The real remanent flux density is chosen to be not only aligned but proportional to the virtual field $\boldsymbol{H}_{2}$.

When the last two conditions are satisfied, the real remanent flux density is both solenoidal and irrotational, thus, satisfying the ideal remanence equations of Ref. [23]. When all the conditions above are satisfied, the magnetic structure in $R_{m}$ is maximally energy efficient.

Within the same framework, it can be shown that the maximally energy-efficient border between the permanent magnet and the highly permeable material is given by a level surface of the magnetic scalar potential $\Phi_{2}$ that generates the virtual field: $\boldsymbol{H}_{2}=-\nabla \Phi_{2}$ [23].

\section{OPTIMAL SEGMENTATION}

We now address the question of how best to segment the solution with a continuously varying remanence into $N$ uniformly magnetized segments. If the border of each segment is predetermined, the optimal remanence direction will just be the direction of the virtual field averaged over the volume of the segment. However, if the shape of the segments is not predetermined, the optimal border of each segment has to be determined from the optimization. We will now consider this problem as a function of the number of segments that the system is desired to be split into.

For simplicity, we consider two-dimensional problems, i.e., systems in which the extent in one dimension is greatly larger than along the other two dimensions, and no physical quantities are varying along this direction. An example is an infinitely long hollow cylinder.

The starting point to the solution of the segmentation problem is the observation that the optimal border between two adjacent segments always lies on a contour line of the direction of the virtual field $\boldsymbol{H}_{2}$. The direction of $\boldsymbol{H}_{2}$ is determined by the angle $\psi=\arctan \left(H_{y} / H_{x}\right)$, where $H_{x}$ and $H_{y}$ are the two components of the virtual field $\boldsymbol{H}_{2}$. The optimality of the contour lines of $\psi$ is a consequence of the independence between the contributions to the value of $\mathcal{S}$ from different points of the magnet. The derivation is given in Appendix A; intuitively, since the contribution from a point of the magnet is proportional to the scalar product between the virtual field in that point and the remanence of the real system, the orientation of the virtual field is the only relevant variable. A given point of the magnet is best assigned to the segment which gives the best match between the direction of the virtual field $\boldsymbol{H}_{2}$ and the remanence of the real magnet $\boldsymbol{B}_{r 1}$. For the example of the Halbach cylinder of infinite length, the contour lines of $\psi$ are coincident with the contour lines of the angular coordinate $\phi$.

This observation considerably reduces the search space of the optimization problem: we are left with the simpler problem of determining which of the contour curves must be selected as borders between segments. Answering this question is equivalent to finding the best piecewise linear approximation to a continuous curve, such that the perimeter of the piecewise curve is maximized. We start by illustrating the approach with a simple example before stating the general conditions for this equivalence to be true. Let us consider again the example of the Halbach cylinder and the corresponding optimal remanence $\boldsymbol{B}_{r 1}$ given by Eq. (5). As the angular coordinate $\phi$ goes from 0 to $2 \pi$, the vector $\boldsymbol{B}_{r 1} \propto \boldsymbol{H}_{2}$ performs two complete revolutions. Instead of considering $\arctan \left(H_{y} / H_{x}\right)$, which has values in the interval $[-\pi,+\pi]$, we can introduce the accumulated angle which, in this case, is equal to $2 \phi$, and assumes the values in the interval $[0,4 \pi]$. Below, we use $\psi$ for this accumulated angle.

Once a starting value $\psi_{0}$ is fixed, it is possible to define the parametrized curve $\mathcal{H}\left(\psi_{1}\right)$ as

$$
\mathcal{H}\left(\psi_{1}\right)=\int_{\mathcal{R}_{\left[\psi_{0}, \psi_{1}\right]}} d^{2} x \boldsymbol{H}_{2}(\boldsymbol{x})
$$

The curve has the dimension of magnetic field integrated over an area. The integration domain $\mathcal{R}_{\left[\psi_{0}, \psi_{1}\right]}$ is a subset of the design area $R_{m}$ defined as

$$
\mathcal{R}_{\left[\psi_{0}, \psi_{1}\right]}=\left\{\boldsymbol{x}: \psi(\boldsymbol{x}) \in\left[\psi_{0}, \psi_{1}\right]\right\} \cap R_{m} .
$$

As shown in Appendix B, for the continuous case, the value of the objective functional $\mathcal{S}$ is given by the length of the curve $\mathcal{H}(\psi)$. For the segmented case with splitting angles $\left\{\psi_{j}\right\}_{j=0, \ldots, N}$, the value of $\mathcal{S}$ reduces to the length of the piecewise linear curve inscribed in $\mathcal{H}(\psi)$ :

$$
\begin{aligned}
\mathcal{S}_{\text {continuous }} & =\int_{\psi_{0}}^{\psi_{0}+4 \pi}\left\|\frac{d}{d \psi} \mathcal{H}(\psi)\right\| d \psi \rightarrow \mathcal{S}_{\text {segmented }}^{(N)} \\
& =\sum_{j=0}^{N-1}\left\|\int_{\psi_{j}}^{\psi_{j+1}} \frac{d}{d \psi} \mathcal{H}(\psi) d \psi\right\|
\end{aligned}
$$

For the case of the Halbach cylinder, the curve $\mathcal{H}(\psi)$ is given by two revolutions around a point. This implies that segmenting the Halbach magnet into $N$ pieces will result in a reduction of the value of $\mathcal{S}$ according to the familiar formula [21]:

$$
\frac{\mathcal{S}_{\text {segmented }}^{(N)}}{\mathcal{S}_{\text {continuous }}}=\frac{N \sin (2 \pi / N)}{2 \pi} .
$$

Any other possible way to segment this volume using the same number of segments results in a greater reduction. As an example, we consider a segmentation using seven pieces. The curve $\mathcal{H}(\psi)$ and the inscribed seven-segment piecewise linear approximation for the Halbach example are shown in Fig. 2(a), and the resulting optimal segmentation is shown in Fig. 2(b). Because of the symmetry, the choice of the starting point $\psi_{0}$ indicated in Figs. 2(a) and 2(b) as a black dot does 


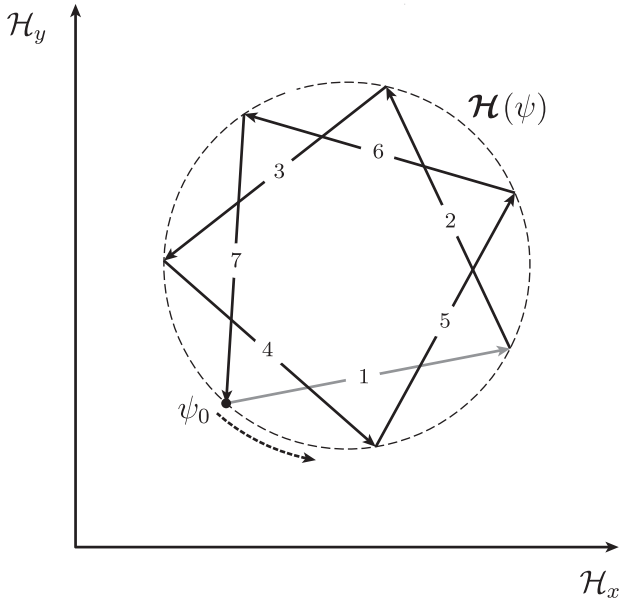

(a)

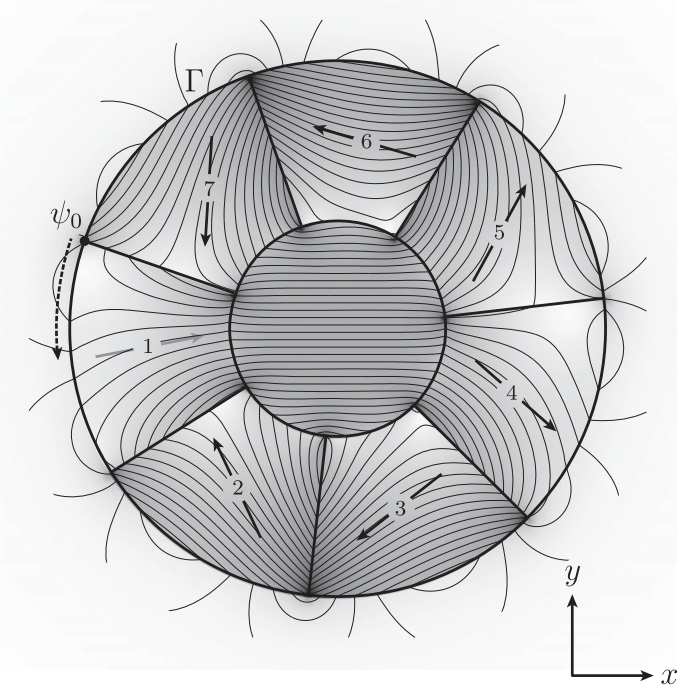

(b)

FIG. 2. Optimal segmentation of the Halbach cylinder geometry using seven segments. Because of the symmetry, the starting point indicated in both the panels by a black dot does not affect the value of the objective functional. (a) The curve $\mathcal{H}(\psi)$ and its segmentation. (b) Corresponding segmented geometry.

However, while this condition is conjectured to be sufficient, it does not exhaust the class of design objectives for which the globally optimal segmentation can be determined using our approach.

When the union of all the $\psi$ contour lines intercepting $\Gamma$ spans the whole design area, it is possible to reduce the problem of optimal segmentation to the problem of approximating the continuous curve $\mathcal{H}(\psi)$ with a piecewise linear curve so that the length is maximized. This fact is shown in Appendix B. The globally optimal solution to this problem can be determined employing dynamic programming [24].

If the curve $\Gamma$ is closed, as it is in the Halbach example, the choice of the starting value $\psi_{0}$ can affect the value of $\mathcal{S}$ : it is still possible to determine the dependence of $\mathcal{S}$ on the starting point and find the optimal value of $\psi_{0}$. However,

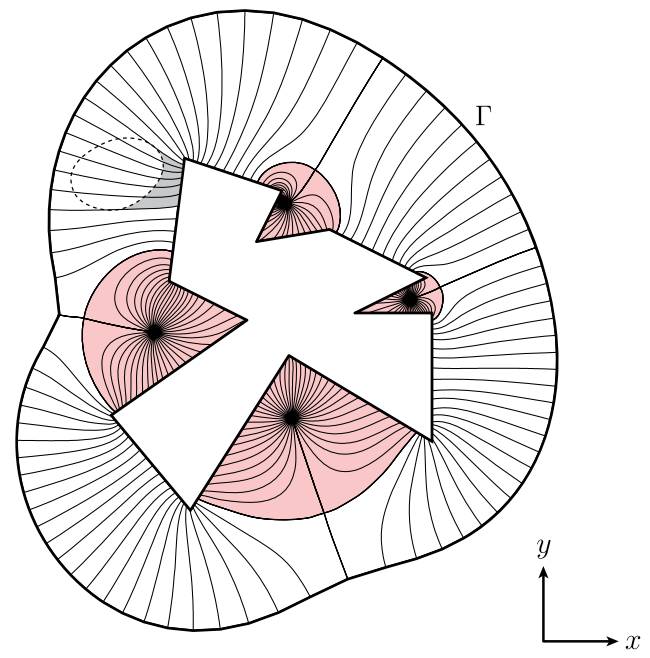

FIG. 3. Example of a geometry for which the $\psi$ contour lines passing by the curve $\Gamma$ do not span the whole design area. As explained in Sec. III, the globally optimal segmentation cannot be determined by our method for this case. 
for the case of the Halbach cylinder with optimal external border, all the starting points are equivalent.

If the design area is not connected, each of the disconnected regions can be segmented independently using the same method. If the design area is not entirely spanned by the $\psi$ contour lines passing by $\Gamma$, the picture is more complex, and this algorithm cannot determine the globally optimal solution. However, it remains true that the optimal borders between segments have to be contour lines of $\psi$. Moreover, it is still possible to find the optimal segmentation with respect to a limited search space.

Hitherto, we consider only two-dimensional systems. However, it is also possible to analyze the problem of segmenting a given design region $R_{m}$ belonging to a threedimensional system. It remains true that it is not optimal to split a region over which the direction of the virtual field is constant. This leaves 2 degrees of freedom such as the values of the azimuthal angle $\psi=\arctan \left(H_{y} / H_{x}\right)$ and the inclination angle $\vartheta=\arccos \left(H_{z} /\|\boldsymbol{H}\|\right)$ of the virtual field $\boldsymbol{H}_{2}$. In this case, the problem consists of subdividing a region of the $\psi-\vartheta$ plane into simply connected subsets. The globally optimal solution to this problem cannot be determined using the same techniques that have been described for the two-dimensional case. It is still possible to approach a three-dimensional problem by considering the procedure described in Sec. V.

In the present section, we derive the framework to solve the problem of maximizing a linear functional with respect to the geometrical subdivision of a certain design area into a given number of uniformly magnetized segments. Since the value of the objective increases monotonically with the number of segments, approaching the limit given by the continuous case, the optimization problem is equivalent to minimizing the number of segments with a given value of the objective. The reason is that generally the manufacturing cost of a magnetic system increases with the number of subdivisions of the same volume of permanent magnet. However, the realization of segments whose borders are curved surfaces might be challenging and drive up the costs. One possible solution is to approximate the optimal shapes predicted by the theoretical approach with simplified geometries that are more feasible to produce. Another possibility is to consider the procedure described at the end of Appendix B, which has the purpose of constraining the family of possible segmentations to predefined shapes.

\section{EXAMPLES}

The procedure outlined in Sec. III is now illustrated with different examples associated with figures showing the result of the finite-element-method simulation of the optimized configuration. The remanence always has the same norm for all the magnet segments, and its direction is indicated in the figures by the black arrows. The norm of the magnetic flux density $\boldsymbol{B}_{1}$ is indicated in gray scale, darker shades corresponding to a higher value of the norm. The field lines of $\boldsymbol{B}_{1}$ are shown as thin black lines.

\section{A. Halbach cylinder}

We now examine in detail the case of the Halbach cylinder, which we discuss briefly in Sec. II.

We adopt cylindrical coordinates $r$ and $\phi$, and we denote the unit vectors in the radial and azimuthal directions by $\hat{\boldsymbol{e}}_{r}$ and $\hat{\boldsymbol{e}}_{\phi}$, respectively. The field $\boldsymbol{H}_{2}$ generated outside an infinitely long cylinder magnetized transversally to its axis is given by

$$
\boldsymbol{H}_{2}(r, \phi)=\frac{M R_{I}^{2}}{r^{2}}\left[\cos (\phi) \hat{\boldsymbol{e}}_{r}+\sin (\phi) \hat{\boldsymbol{e}}_{\phi}\right],
$$

where the axis of the cylinder is in the $z$ direction, the magnetization is in the $x$ direction, $R_{I}$ is the radius of the cylinder, and $M$ the norm of its magnetization. This cylinder is the virtual magnet for the Halbach case.

The norm of the virtual magnetic field $\mathrm{H}_{2}$ is independent of $\phi$, and the level curves of the norm are circles with their center in the origin. One of these circles is chosen as the external border. The points of the external border can be parametrized directly by the angle $\phi \in[0,2 \pi]$, and the orientation of the virtual field is described by the accumulated angle $\psi=2 \phi \in[0,4 \pi]$. The radius of the external border is denoted by $R_{O}$. The level curves of $\psi$ are radial lines lines with constant $\phi$. The vector $\mathcal{H}_{\left[\psi_{0}, \psi_{1}\right]}$ can be calculated explicitly:

$$
\begin{aligned}
\mathcal{H}_{\left[\psi_{0}, \psi_{1}\right]}= & \int_{\mathcal{R}_{\left[\psi_{0}, \psi_{1}\right]}} d^{2} x \boldsymbol{H}_{2}(\boldsymbol{x}) \\
= & \left(M R_{I}^{2}\right) \int_{\psi_{0} / 2}^{\psi_{1} / 2} d \phi\left(\cos (\phi) \hat{\boldsymbol{e}}_{r}\right. \\
& \left.+\sin (\phi) \hat{\boldsymbol{e}}_{\phi}\right) \int_{R_{I}}^{R_{O}} d r \frac{1}{r}, \\
= & \left(M R_{I}^{2}\right) \log \left(\frac{R_{O}}{R_{I}}\right)\left(\hat{\boldsymbol{e}}_{x} \int_{\psi_{0} / 2}^{\psi_{1} / 2} d \phi \cos (2 \phi)\right. \\
& \left.+\hat{\boldsymbol{e}}_{y} \int_{\psi_{0} / 2}^{\psi_{1} / 2} d \phi \sin (2 \phi)\right), \\
= & \left(M R_{I}^{2}\right) \log \left(\frac{R_{O}}{R_{I}}\right) \frac{1}{2}\left(\hat { \boldsymbol { e } } _ { x } \left[\sin \left(\psi_{1}\right)\right.\right. \\
& \left.\left.-\sin \left(\psi_{0}\right)\right]-\hat{\boldsymbol{e}}_{y}\left[\cos \left(\psi_{1}\right)-\cos \left(\psi_{0}\right)\right]\right) .
\end{aligned}
$$

Since $\psi \in[0,4 \pi]$, the curve $\mathcal{H}(\psi)=\mathcal{H}_{\left[\psi_{0}, \psi\right]}$ describes two revolutions around the point:

$$
\left(-\sin \left(\psi_{0}\right) \hat{\boldsymbol{e}}_{x}+\cos \left(\psi_{0}\right) \hat{\boldsymbol{e}}_{y}\right)\left(M R_{I}^{2}\right) \log \left(R_{O} / R_{I}\right) / 2
$$

with radius $R_{B}=\left(M R_{I}^{2}\right) \log \left(R_{O} / R_{I}\right) / 2$. The initial point is in the origin: $\mathcal{H}\left(\psi_{0}\right)=\mathbf{0}$. 
The length of this curve in the continuous case is equal to the value of $\mathcal{S}$ and is given by $4 \pi R_{B}$. Intuitively, since the curvature of the curve is constant, the best way to split it in $N$ segments in order to maximize the length of the polygonal line is to use identical segments. If this curve is segmented using $N$ identical segments (for the two revolutions), each segment will span an angle of $(4 \pi) / N$. The side $s$ of a regular polygon with $N$ sides inscribed in a polygon of radius $R$ is equal to $s=2 R \sin (\pi / N)$. Considering the double revolution, the ratio between the length of the vector $\mathcal{H}$ associated with each segment and the length of the corresponding curve arc is given by

$$
\frac{\mathcal{S}_{N}}{\mathcal{S}_{\text {continuous }}}=\frac{N \sin (2 \pi / N)}{2 \pi},
$$

which is the familiar formula for the segmented Halbach cylinder [21].

The $\mathcal{H}(\psi)$ curve and the geometry are shown in Figs. 2(a) and 2(b) for the case $N=7$.

It is worth mentioning that the results of this section are also true for any multipole Halbach cylinder surrounded by air. This is shown by considering the analytical expression of the field generated by a multipole Halbach cylinder [25]. If the virtual remanence is defined over an infinitely long cylindrical shell $R_{g}$ and is expressed as $\boldsymbol{B}_{r 2}(r, \phi)=$ $B_{r}(r)\left[\cos (+p \phi) \hat{\boldsymbol{e}}_{r}+\sin (+p \phi) \hat{\boldsymbol{e}}_{\phi}\right]$, then the optimal real remanence is oriented as $\cos (-p \phi) \hat{\boldsymbol{e}}_{r}+\sin (-p \phi) \hat{\boldsymbol{e}}_{\phi}$. The design area is adjacent to $R_{g}$ and is located on the external side of $R_{g}$ if $p<0$ and on the internal side of $R_{g}$ if $p>0$. The optimal external border of the design area is always a cylinder which is coaxial with $R_{m}$. Moreover, the optimal boundaries between adjacent segments are given by radial surfaces, all separated by equal angles. The ratio between the value of $\mathcal{S}$ in the segmented case and the value in the continuous case is still given by Eq. (16). In the continuous case, the field-generated $\boldsymbol{H}_{1}$ generated by the real remanence has exactly the same angular dependence as the virtual remanence:

$\boldsymbol{H}_{1} \propto r^{p-1}\left[\cos (+p \phi) \hat{\boldsymbol{e}}_{r}+\sin (+p \phi) \hat{\boldsymbol{e}}_{\phi}\right]$ for $p \neq 1$

The case analyzed above of uniform field in the $x$ direction is given by $p=-1$, and the region $R_{g}$ is a cylinder instead of a hollow cylindrical shell.

\section{B. Rectangular cavity}

A rectangular cavity is another example for which the equation of the virtual field is analytically solvable. In order to show the algorithm's results with an asymmetrical problem, a decentered circle is chosen as the external border of the design area, as is visible in Fig. 4(b).

The virtual remanence is uniform in the cavity in direction $\hat{\boldsymbol{e}}_{y}$. The accumulated angle $\psi$ spans an angle of $4 \pi$ as in the previous example. This implies that the curve $\mathcal{H}(\psi)=\mathcal{H}_{[\psi, \psi \psi}$ can be extended indefinitely on both sides according to

$$
\mathcal{H}(\psi+4 \pi n)=\mathcal{H}(\psi)+n \mathcal{H}_{\left[\psi_{0}, \psi_{0}+4 \pi\right]},
$$

where $\mathcal{H}_{\left[\psi_{0}, \psi_{0}+4 \pi\right]}$ is the integral of the virtual field over the whole design region. The curve $\mathcal{H}(\psi)$ is plotted in Fig. 4(a) over a period of $8 \pi$. Selecting a different starting point moves the origin of the $\mathcal{H}_{x}-\mathcal{H}_{y}$ plane indicated as a black dot on different points of the curve $\mathcal{H}(\psi)$. Any interval $\left[\psi_{0}, \psi_{0}+4 \pi\right]$ can be used for the segmentation corresponding to the different starting points on the external border of the magnet. The optimal position of the point $\psi_{0}$

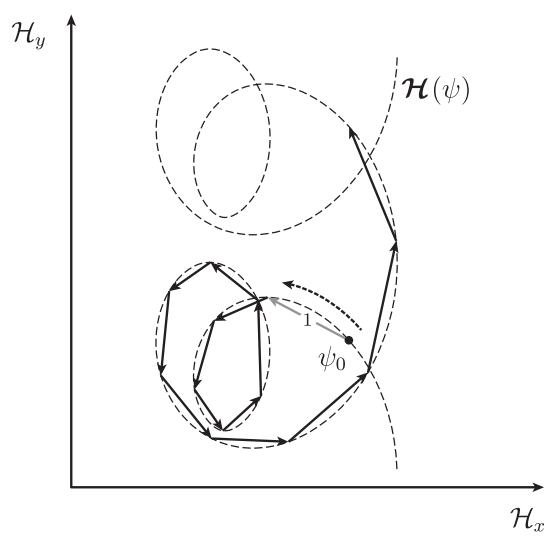

(a)

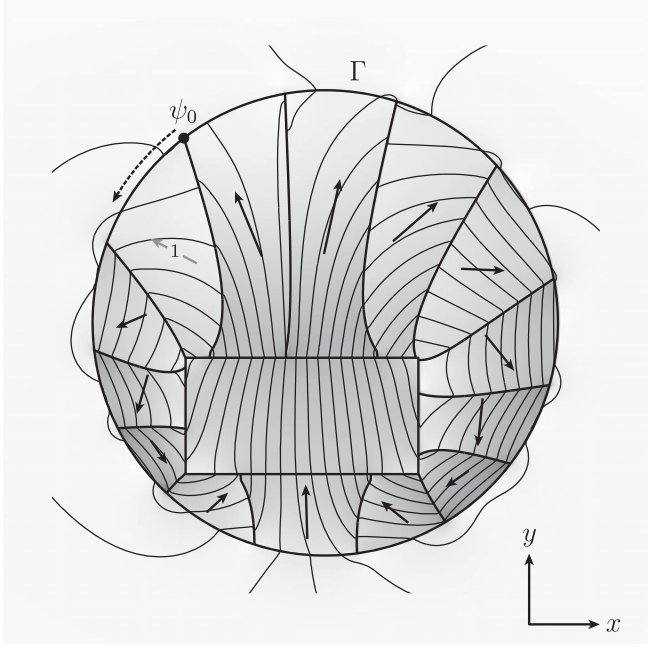

(b)
FIG. 4. The segmentation of a circular design region with a decentered rectangular cavity is shown in (b). The objective functional $\mathcal{S}$ is the $y$ component of the field integrated over the rectangular air gap. The curve $\mathcal{H}(\psi)$ and its optimal segmentation are shown in (a) as, respectively, a dashed curve and a collection of adjacent arrows. The arrows represent the optimal segmentation of the curve, their direction indicates the optimal direction of the remanence in each segment, and their length is proportional to the contribution to the value of $\mathcal{S}$ from each segment. The norm of the remanence is the same for all segments. 
for $N=14$ segments is determined numerically from $\mathcal{H}(\psi)$ with a separate optimization step. The point $\mathcal{H}\left(\psi_{0}\right)$ is indicated by the black dot in Fig. 4(a) and corresponds to the point of the external border indicated in Fig. 4(b). The vectors $\mathcal{H}$ resulting from the optimal segmentation are indicated as arrows in both the panels; the gray arrow indicates the direction of $\mathcal{H}$ for the first segment.

\section{Systems including iron}

We now consider two examples which also involve highpermeability material. For these systems, we use FEM calculations to compute the virtual field. In both the cases pictured in Figs. 5(a) and 5(b), the position of the iron yokes and core is predetermined, but the border between hard magnets and air is optimal with the given volume constraint and so are the borders between the different segments. The parts of the geometry corresponding to the iron are represented as hatched areas.

The objective in the example of Fig. 5(a), which is a model of a permanent-magnet electric motor, is to create a quadrupole field directed radially and located in the air gap between the magnets and the external iron yoke. The virtual remanence is expressed in cylindrical coordinates as $\boldsymbol{B}_{r 2}(\rho, \phi)=\hat{\boldsymbol{e}}_{\rho} \sin (2 \phi)$. The design area is the region between the internal border of the air gap and the external border of the iron core. To illustrate how the optimal border between iron and permanent magnet can be found, we put an arbitrary constraint on the magnet volume. In this example, we arbitrarily require that the magnet occupies $80 \%$ of the original design area and that the remaining part is filled with air. We then calculate the optimal border between magnet and air that is consistent with this constraint. The result is that the four regions adjacent to the inner iron core are excluded from the design area, as is visible in Fig. 5(a). Even though the objective of the optimization algorithm is the intensity of the field rather than its quality, the result of this example reproduces the desired field shape quite accurately.

In case of Fig. 5(b), the objective is, again, as in the yokeless Halbach example, to maximize the field in the $x$ direction averaged over the inner circular cavity. In this case, the design area is the region between the internal border of the iron yoke and the external border of the circular cavity. We arbitrarily require that the magnet occupies $70 \%$ of the original design area. The remaining part is then filled with air, and the optimization of the border between magnet and air determines the shape of the two empty regions that are visible in Fig. 5(b). Despite the fact that the virtual magnet of this example is identical to the one of the Halbach cylinder, the virtual field $\boldsymbol{H}_{2}$ is not given by Eq. (11) for this case, since the presence of the iron yoke affects the solution. The consequence is that the optimal direction of the remanence with respect to the linear objective is different for the two cases and so are the optimal borders between magnets and air and the ones between adjacent segments.

It is insightful to compare the yoked and yokeless cylinders for the ideal case of a remanence field of constant norm but continuously varying direction. Because of its selfinsulation properties, an ideal Halbach cylinder whose remanence field is described by Eq. (5) generates the same field if an iron yoke is introduced at its external border. In the presence of the yoke, the Halbach solution, which is still optimal with respect to the purpose of generating a perfectly uniform field, is not the one producing the highest field intensity, i.e., maximizing the linear objective. Conversely, the field generated by the ideal solution corresponding to the highest intensity is not perfectly homogeneous.

Both examples in this section show the orthogonality between the optimal remanence and border between the magnet and iron. We see that the border between the magnet and air is always normal to the border between two segments. This is a consequence of the virtual field being both solenoidal and irrotational.

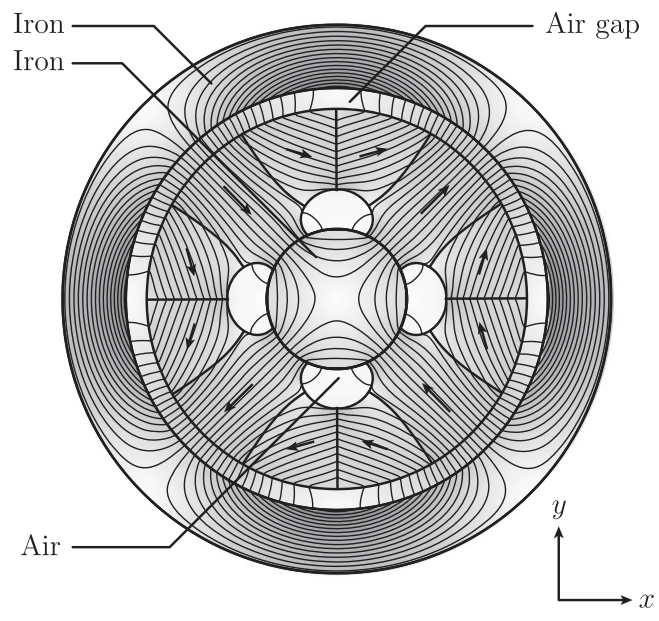

(a)

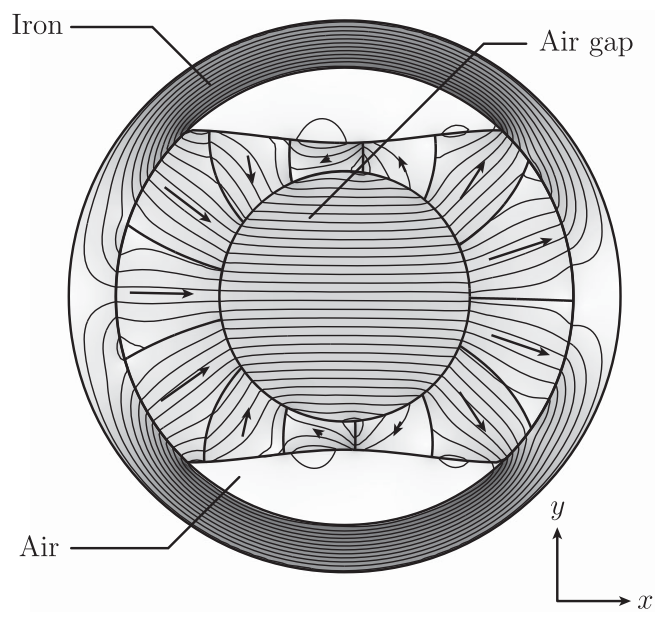

(b)
FIG. 5. Two examples of geometries involving iron parts and a constraint on the amount of magnetic material. (a) Quadrupole motor and (b) Yoked Halbach. In both examples the borders between the magnets and the parts of the design region filled with air have the optimal shape with respect to the constraint on the magnet volume. The arrows indicate the optimal segmentation of the curve $\mathcal{H}(\psi)$; thus, the length of each arrow corresponds to the contribution from that segment to the value of the objective $\mathcal{S}$. 


\section{Comparison with uniform field magnets}

It is instructive to compare the results of our method, which can also be applied to maximize the field along one direction averaged over a closed air gap, with another design approach which starts with the assumption that the field is perfectly homogeneous.

Abele and Rusinek [26-28] developed an elegant framework for the design of segmented magnetic systems which can generate a perfectly uniform field $\boldsymbol{H}_{0}$ inside a closed cavity and are perfectly self-insulated from the surroundings. We consider the results in two-dimensional yokeless magnetic systems composed of a uniformly magnetized prism with triangular cross sections. We assume that the permanentmagnet material has zero susceptibility and that the norm $B_{r}$ of the remanent flux density is constant. Any region of the system which is not occupied by magnet is occupied by air.

The closed cavity is a polygon with $Q$ sides, which may be irregular. A single-layer structure in this framework must be composed of exactly $N=3 Q$ segments. Once the shape of the cavity is fixed, the geometry of the structure is entirely determined by two decisions: the ratio $K=H_{0} / B_{r}$ between the norm of the field inside the cavity and the norm of the remanence and the position of a point $\boldsymbol{x}_{F}$ inside the cavity in which the magnetic scalar potential is zero. The direction of $\boldsymbol{H}_{0}$ determines the direction of the remanence of each segment, but it does not affect the geometry of the system. When the cavity is a regular polygon, the center of the polygon is the position of $\boldsymbol{x}_{F}$ which minimizes the volume $V_{m}$ of magnetic material and leads to the highest efficiency. An example is shown in Fig. 6(a): the cavity is an equilateral triangle, the field $\boldsymbol{H}_{0}$ is oriented in the $y$ direction, and the point $\boldsymbol{x}_{F}$ is in the center of the triangle.

This approach differs from the one presented in this paper since the resulting field is perfectly uniform, but the approach cannot be applied to any other objective, except the generation of a uniform dipole field. Moreover, there is a constraint on the number of segments, and for single-layer structures, the volume of the magnet goes to infinity as $H_{0}$ goes to $B_{r}$. Since both are segmentation algorithms, it is interesting to compare the performance of the two approaches. This is best done by calculating the figure of merit $\mathcal{M}$ introduced in Eq. (6). We consider magnetic assemblies designed to generate a field in the $y$ direction; therefore, we replace $\boldsymbol{B}_{1}$ in the numerator of Eq. (6) with its $y$ component. For the case of a uniform field, this replacement has no effect, but it is a more realistic way to estimate the efficiency of our method, since the field is not perfectly uniform. We also introduce the figure of merit $\eta$ which characterizes the homogeneity of the field and is zero for a uniform field:

$$
\eta=\frac{\left\langle B_{1}^{2}\right\rangle-\left\langle B_{1}\right\rangle^{2}}{\left\langle B_{1}^{2}\right\rangle} .
$$

The efficiency and uniformity with the two approaches are compared in Figs. 7(a) and 7(b) for different values of magnet volume $V_{m}$ and for different shapes of the inner cavity: an equilateral triangle $Q=3$ and octagon $Q=8$ indicated by red and blue lines, respectively. We also consider a rectangular cavity $Q=4$ with a width that is double the height, indicated by the green lines. The results are plotted as a function of the ratio $V_{m}: V_{g}$ between the volume of the magnet and the volume of the cavity $V_{g}$. The dashed lines indicate uniform field magnets realized with $N=3 Q$ segments, and the dotted or solid lines indicate the magnets optimized for field average using our approach. Since within our framework it is possible to choose of the number of segments, we consider the cases $N=3 Q$ and $N=2 Q$ indicated by the solid lines and the dotted lines, respectively. The efficiency of the Halbach cylinder with continuously varying remanence is indicated in Fig. 7(a) by the black dash-dot line.

Even if $\mathcal{M}$ is not equivalent to the objective functional $\mathcal{S}$ of our optimization approach, the results of this method

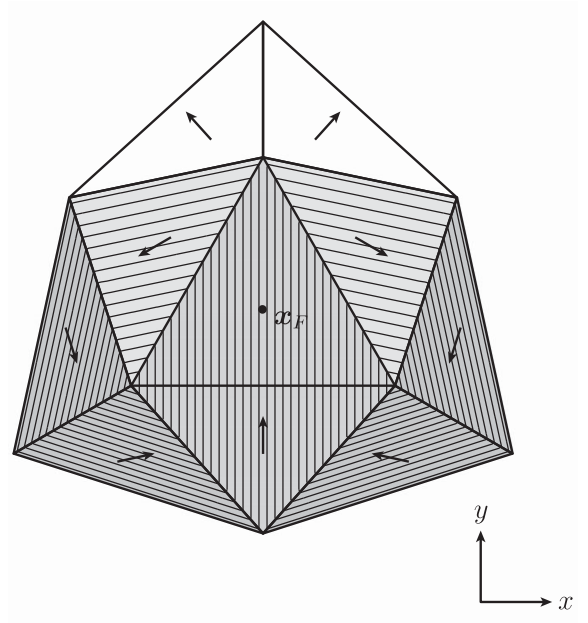

(a)

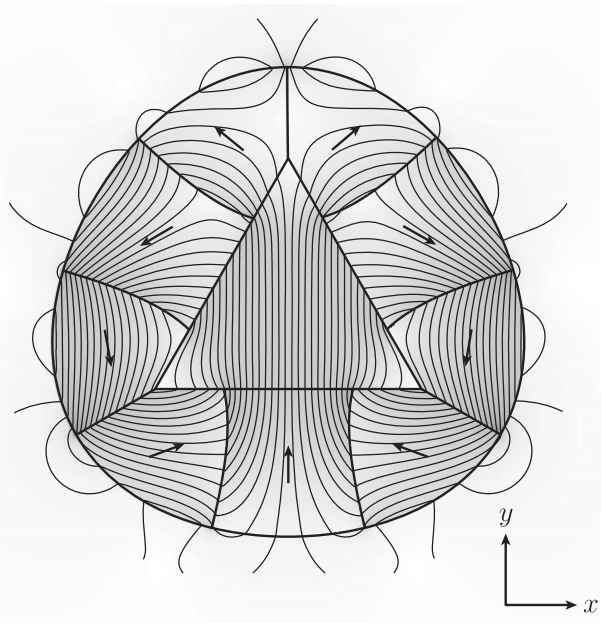

(b)
FIG. 6. Comparison between the two possible ways to segment the magnetic system designed to generate a field in the central triangular cavity and oriented in the $y$ direction. (a) The segmented system which creates a perfectly uniform field inside the cavity. (b) The segmentation determined using our method, which maximizes the $y$ component of the field averaged over the central cavity. The volume $V_{m}$ of magnetic material is the same in the two cases. The arrows indicate the remanence inside each segment. The configuration of (a) produces a field that is also uniform inside each magnet segment. In the two blocks that are on top, the flux density is zero since $\mu_{0} \boldsymbol{H}_{1}=-\boldsymbol{B}_{r 1}$. 


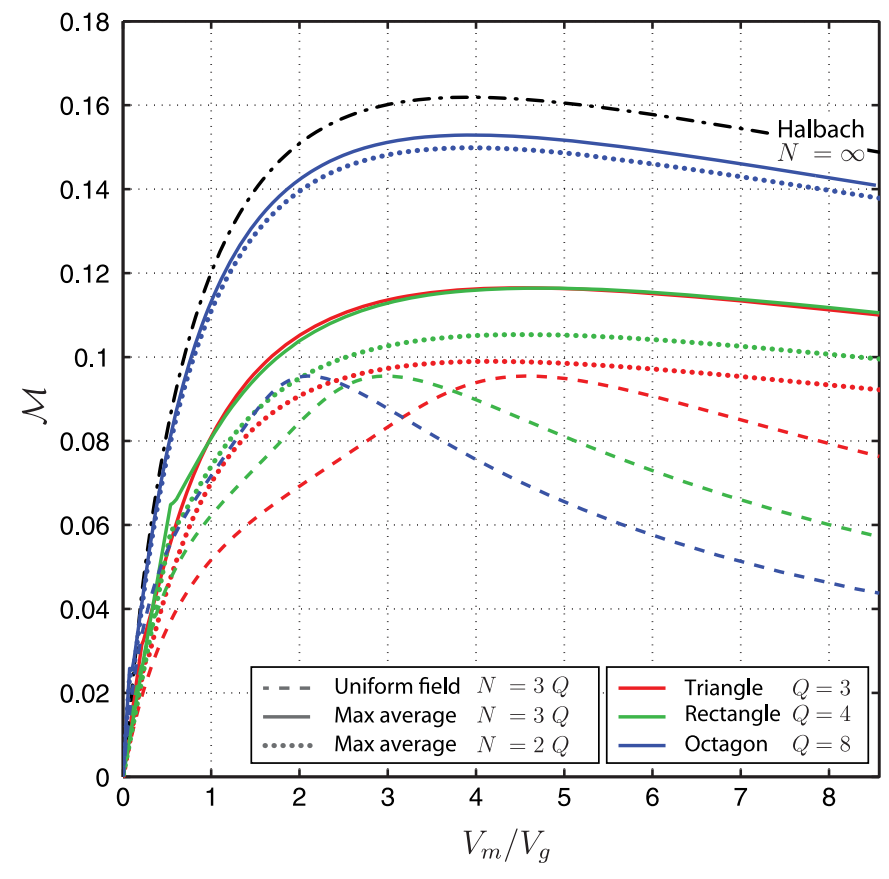

(a)

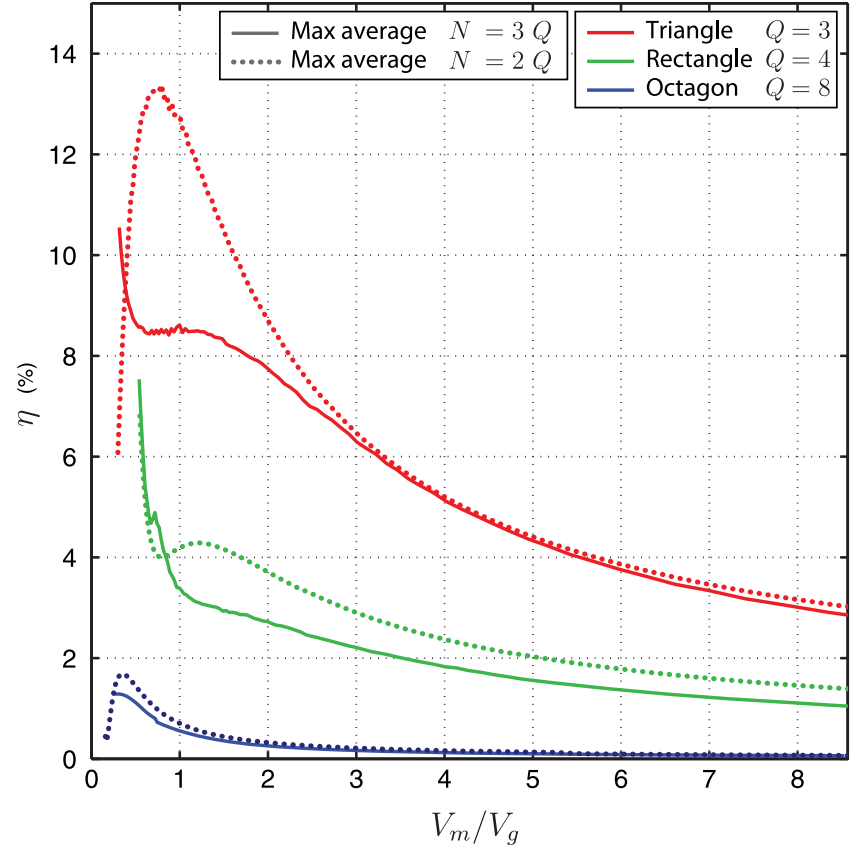

(b)

FIG. 7. Comparison between the performance of the two segmentation approaches for different shapes of the inner cavity. (a) The value of the efficiency figure of merit $\mathcal{M}$. (b) The value of the homogeneity figure of merit $\eta$. In both panels, the results are plotted as a function of the ratio $V_{m}: V_{g}$.

are characterized by a high efficiency when compared to the uniform field designs, even with a smaller number of segments. The choice of a magnet volume higher than the optimal, which results in higher values of field, does not decrease the efficiency with our method as it does for single-layer uniform field magnets.

The uniformity figure of merit $\eta$ is shown in Fig. 7(b). The field is more uniform for the case of the rectangle than it is for the triangle and even more uniform for the case of the octagon. In all cases, the value of $\eta$ is $\lesssim 5 \%$ for magnet volumes above the maximum efficiency $\mathcal{M}$. The data points corresponding to very small magnet volumes are slightly affected by the numerical noise arising from the fact that the calculation of the curve $\mathcal{H}(\psi)$ is performed by a numerical integration. The estimation of the parameter $\eta$ for small volumes is particularly affected by the noise, and, therefore, a few data points are omitted from Fig. 7(b). The limit $V_{m} \rightarrow 0$ is also not very relevant for practical applications since $\mathcal{M} \rightarrow 0$.

The result of the comparison between the two approaches shows the trade-off between field intensity and quality. Whenever the objective vector field $\boldsymbol{u}(\boldsymbol{x})$ is also a physically acceptable magnetic field, the configurations optimized with respect to the linear objective $\mathcal{S}$ can be directly compared with configurations optimized by using other techniques that use as an objective the quality of the field. This class of optimization problems is formulated as the minimization of a nonlinear objective functional such as [19]

$$
\mathcal{D}\left[\boldsymbol{H}_{1}\right]=\left(\int d^{3} x\left\|\boldsymbol{H}_{1}(\boldsymbol{x})-\boldsymbol{u}(\boldsymbol{x})\right\|^{2}\right)^{1 / 2} .
$$

The linear objectives introduced in Sec. II A are suitable for magnet design problems where the main goal is to maximize the average intensity of the field in the direction given by $\boldsymbol{u}(\boldsymbol{x})$ using a fixed amount of permanent-magnet material. This problem is equivalent to minimizing the amount of permanent magnet that is necessary to obtain the same value of $\mathcal{S}$. Even though in many cases our approach is also able to reproduce with a good approximation the desired field shape $\boldsymbol{u}$, the accuracy of the generated field with respect to the required distribution is not the objective of the optimization method presented in this work. The design of magnetic systems for applications that require a very high field precision must be carried out with different techniques. A possibility is to combine the procedure described here with subsequent optimization schemes aimed at correcting small field distortions, such as the ones proposed in Refs. $[8,15,16]$. It is also worth mentioning that the relevance of linear functionals goes beyond those optimization problems that are directly described by this class of objectives, as the theoretical results obtained for linear functionals provide insight into general characteristics of magnet design problems.

\section{THREE-DIMENSIONAL SYSTEMS}

As we mention above, the segmentation procedure does not immediately generalize to three dimensions. 
Nevertheless, one may ask to what extent the segmentation approach can be employed to optimize the segmentation of three-dimensional magnetic systems. The result presented in Appendix A is true for three-dimensional geometries as well: it is not advantageous to subdivide a region of constant direction of the virtual field, that is, a region $R(\vartheta, \psi)$ for which the inclination angle and the azimuthal angle of the virtual field are both constant. As we mention in Sec. III, the additional degree of freedom means that the globally optimal solution must be searched for among all the possible ways of partitioning the $\vartheta-\psi$ plane into a given number of regions. The procedure described in Appendix B does not provide the solution to this problem. On the other hand, applying the one-parameter segmentation procedure to the whole design area is too restrictive for most threedimensional problems. One strategy is to apply the segmentation procedure separately to different parts of the design region identified beforehand. This preliminary decision can be based on considerations about the symmetries exhibited by the geometry of the magnetic system.

For the illustrative examples presented in this section, the symmetry suggests that the design area can be initially subdivided into smaller regions $R_{j}(j=1, \ldots, N)$ separated by level surfaces of the inclination angle $\vartheta$. Our procedure is then applied independently to each of the regions $R_{j}$ to determine the the values of the azimuthal angle $\psi$ that optimize the splitting of $R_{j}$ into $N_{j}$ segments. It must be stressed that the subdivisions between the regions $R_{j}$ and the combinations between the number of segments $N_{j}$ assigned to each region have to be decided beforehand or determined with a separate optimization step.

The geometry of the first example shown in Fig. 8(a) is a three-dimensional generalization of the Halbach cylinder geometry considered in Sec. IVA. The objective is to maximize the $z$ component of the field averaged over the internal spherical cavity. The ideal Halbach sphere with continuously varying remanence produces in the bore a perfectly homogeneous flux density, whose norm is given by [6] $B_{\text {ideal }}=(4 / 3) B_{r} \log \left(R_{O} / R_{I}\right)$, where $R_{O}$ and $R_{I}$ are the external and internal radii of the spherical shell, respectively. The continuously varying remanence field optimized with our approach produces a field that is not perfectly homogeneous, but slightly more intense. The air gap average of the $z$ component of the flux density for the ratio $R_{O}=2 R_{I}$, i.e. the value used in the example, is $B_{\text {continuous }} \approx 1.03 B_{\text {ideal }}$.

We consider all the possible ways to subdivide this geometry into five regions delimited by level surfaces of the inclination angle $\vartheta$ of the virtual field $\boldsymbol{H}_{2}$. Since $\boldsymbol{H}_{2}$ is the field generated by a uniformly magnetized sphere, the knowledge of the analytical solution allows us to take advantage of the symmetries to simplify the procedure. This means that we are able to consider all the possible combinations of number of segments $N_{j}$ for each region and all the possible values of $\vartheta$ determining the subdivisions between these regions. The calculations show that the maximum value of the average air-gap flux density for this family of segmentations corresponds to $0.945 B_{\text {continuous }}$.

Figure 8(a) shows the segmentation corresponding to the best combinations of the parameters for a total number of segments equal to $N_{\text {tot }}=17$. Some of the magnet segments are not displayed in the picture; moreover, some of them are bisected by the plane $y=0$, and the blue surfaces indicate internal cross sections of the bisected blocks, while the gray surfaces are the boundaries of the segments. Each of the two symmetrical polar regions is subdivided into three identical segments. Because of the symmetry, it is not advantageous to subdivide the equatorial region, as all the resulting segments will be magnetized in the $z$ direction. Each of the two symmetrical regions between the poles and the equator is subdivided into five identical segments. The average air-gap flux density for this segmentation is $0.889 B_{\text {continuous }}$.

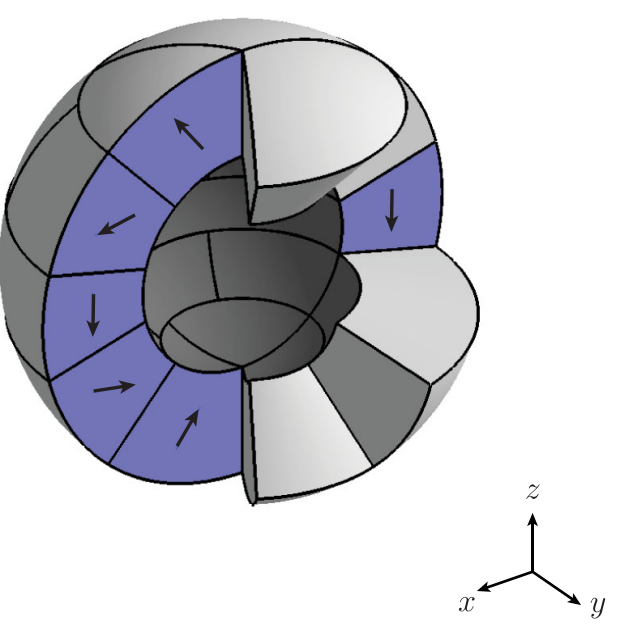

(a)

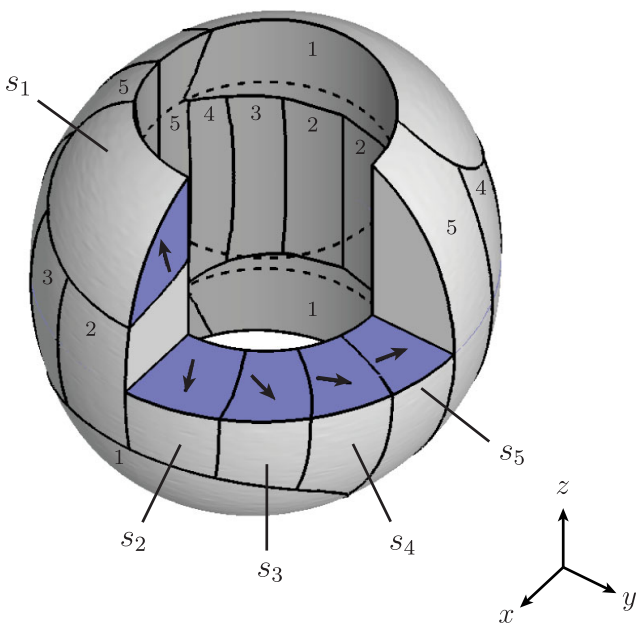

(b)
FIG. 8. Three-dimensional segmented magnetic structures optimized with respect to the average field inside the air gap. (a) Halbach sphere. The field is in the $z$ direction, and the number of segments is $N_{\text {tot }}=17$. (b) Finite-length Halbach cylinder. The field is in the $x$ direction, and the number of segments is $N_{\text {tot }}=20$. 
The second example shown in Fig. 8(b) analyzes how the Halbach cylinder design can be modified when the effects of finite length in the $z$ direction are taken into account. The objective is to maximize the $x$ component of the field averaged over the region of the internal cylindrical cavity $R_{g}$ that is delimited by the two circles indicated by the black dashed lines. Since for many applications it is desirable that the cavity is accessible from the top and from the bottom, the magnet design region extends only on the sides of the cylindrical cavity.

The figure represents a section of the geometry where the first octant is not displayed. Again, the blue surfaces represent sections cutting some magnet block in the middle, while the gray surfaces are all external boundaries of the blocks. As indicated by annotations on the graph, the magnet area is subdivided into five unique shapes denoted by $s_{1}, \ldots, s_{5}$. There are four copies of the shape $s_{1}$, one for each quadrant of the $x-z$ plane, and four copies of each of the shapes $s_{2}, \ldots, s_{4}$, one for each quadrant of the $x-y$ plane. The total number of segments is, thus, $N_{\text {tot }}=20$.

The external border of the magnet is a level surface of $H_{2}$. Segments $s_{1}$ are delimited by level surfaces of $\vartheta$, and the interfaces between segments $s_{2}, \ldots, s_{4}$ are level surfaces of $\psi$. The regions occupied by each of the segments $s_{1}$ could have been subdivided as well, using levels of $\psi$; however, as the direction of the virtual field is quite homogeneous over each of these regions, the advantage of this subdivision would have been minor. The $x$ component of the flux density averaged over the air gap $R_{g}$ is $5 \%$ greater for the magnetic system of Fig. 8(b) than it is for a conventionally segmented Halbach cylinder having the same magnet volume and the same number of segments $N_{\text {tot }}$.

It is noticed that the portion of the magnet area extending above the dashed lines is mainly occupied by segments $s_{1}$ and $s_{5}$. This part of the magnet area can be thought of as an additional structure that can be attached to the conventional Halbach cylinder geometry in order to reduce the detrimental border effects and presents similarities with the structure proposed in Ref. [29].

\section{CONCLUSION}

In conclusion, we present an approach which automatically gives the optimal design of magnetic systems with respect to any linear objective functional. For the case of hard magnets with zero susceptibility, the approach provides the globally optimal shape of the external border between magnet and air with a given volume constraint. The method also gives the globally optimal segmentation of two-dimensional systems with a given number of uniformly magnetized segments. This approach is versatile and being based on an analytical framework, it can be implemented into a fast and efficient algorithm, and the optimality is guaranteed.

\section{ACKNOWLEDGMENTS}

This work is financed by the ENOVHEAT project which is funded by Innovation Fund Denmark (Contract No. 12132673).

\section{APPENDIX A: Optimal segment borders}

In this section, we show that an optimal segmentation consists of segments whose mutual borders are lines of constant direction of the virtual field. This we do by showing that any segmentation which has a curve section $R(\psi)$ of constant $\psi$ divided between two segments can always be replaced by a segmentation which assigns $R(\psi)$ completely to one segment and which is at least as good as the original segmentation.

We start with the objective functional $\mathcal{S}$ defined in Eq. (3) in terms of the field $\boldsymbol{H}_{1}$ and expressed in terms of the remanence $\boldsymbol{B}_{r 1}$ as

$$
\mathcal{S}\left[\boldsymbol{H}_{1}\right]=\int d^{2} x \boldsymbol{B}_{r 1}(\boldsymbol{x}) \cdot \boldsymbol{H}_{2}(\boldsymbol{x}),
$$

where $\boldsymbol{H}_{2}$ is the virtual field. The optimization for the continuous case is immediate: we simply choose a remanence which in all points of the design region is aligned with $\boldsymbol{H}_{2}$. Assuming that the remanence is constant in magnitude (and setting this magnitude equal to unity for convenience), we get the optimized value of the functional:

$$
\mathcal{S}_{\text {continuous }}=\int_{R_{m}} d^{2} x\left\|\boldsymbol{H}_{2}(\boldsymbol{x})\right\| .
$$

We now consider the segmentation of the design area $R_{m}$ into $N$ segments. We introduce the characteristic functions $\phi_{n}(\boldsymbol{x})$ that determine to which segment the point $\boldsymbol{x}$ is assigned. They can assume only the values 0 or 1 . If a given point $\boldsymbol{x}$ is assigned to the $k$ th segment, $\phi_{k}(\boldsymbol{x})=1$ while $\phi_{n \neq k}(\boldsymbol{x})=0$. The objective functional $\mathcal{S}$ corresponding to the segmentation $\left\{\phi_{n}\right\}$ is then given by

$$
\mathcal{S}\left[\left\{\phi_{n}\right\}\right]=\sum_{n=1}^{N} \boldsymbol{B}_{r 1}(n) \cdot \int d^{2} x \phi_{n}(\boldsymbol{x}) \boldsymbol{H}_{2}(\boldsymbol{x}),
$$

since $\boldsymbol{B}_{r 1}(n)$ is constant on segment $n$. Now, the direction of the optimal remanence $\boldsymbol{B}_{r 1}^{\text {opt }}(n)$ for the $n$th segment is clearly aligned with $\int d^{2} x \phi_{n}(\boldsymbol{x}) \boldsymbol{H}_{2}(\boldsymbol{x})$. As before, we can then write $\mathcal{S}$ as

$$
\mathcal{S}\left[\left\{\phi_{n}\right\}\right]=\sum_{n=1}^{N}\left\|\int d^{2} x \phi_{n}(\boldsymbol{x}) \boldsymbol{H}_{2}(\boldsymbol{x})\right\| .
$$

Thus, the problem of finding an optimal segmentation is equivalent to finding a set of characteristic functions $\left\{\phi_{n}\right\}$ which maximize the value of $\mathcal{S}$ in Eq. (A4).

To proceed we make a change of integration variables. Each set $R\left(\psi_{a}\right)=\left\{\boldsymbol{x}: \psi(\boldsymbol{x})=\psi_{a}\right\} \cap R_{m}$ defined by a particular value of $\psi_{a}$ is a finite-length segment of a curve of 


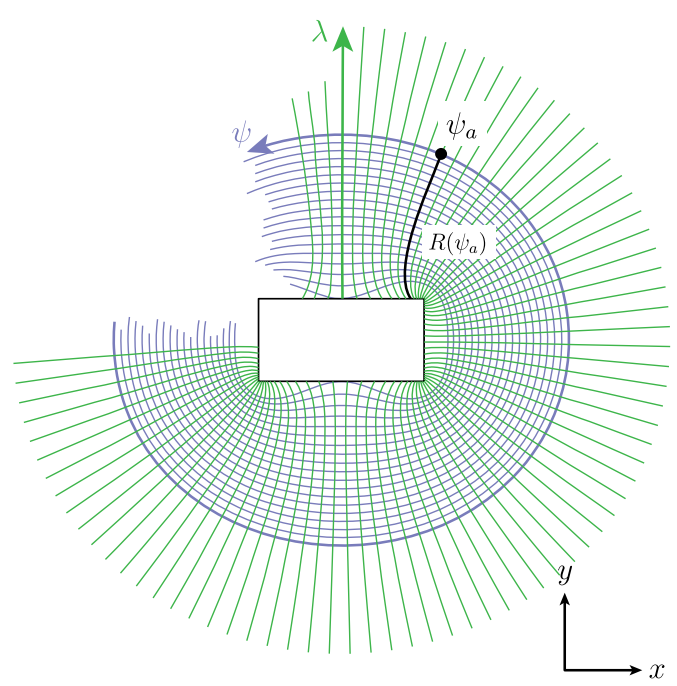

(a)

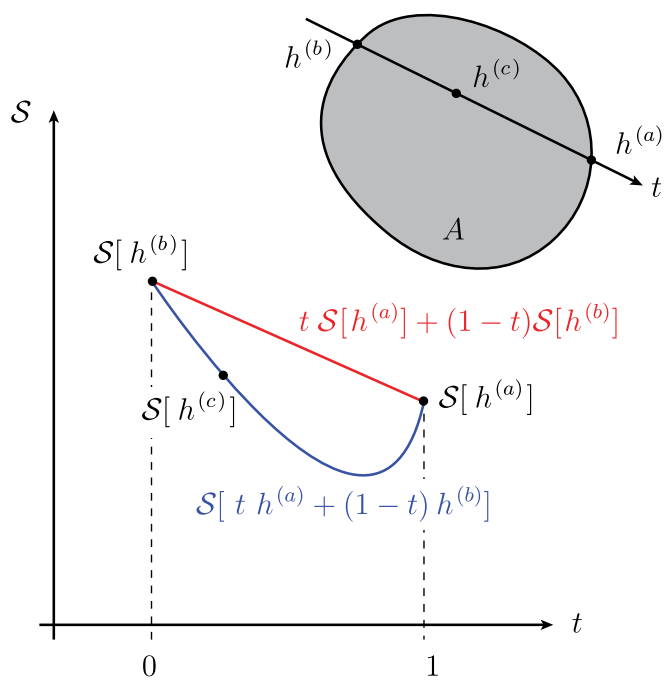

(b)
FIG. 9. (a) The coordinate transformation $\boldsymbol{x} \rightarrow(\psi, \lambda)$ is illustrated on an example geometry. (b) A convex set $A$ and the value of the convex function $\mathcal{S}$ over a line segment traced between two points $h_{n}^{(a)}(\psi)$ and $h_{n}^{(b)}(\psi)$ belonging to the boundary of $A$, illustrating that either $\mathcal{S}\left[h^{(a)}\right]$ or $\mathcal{S}\left[h^{(b)}\right]$ is greater than or equal to the value of $\mathcal{S}$ for any other point on the line segment. constant direction of the virtual field; it can be parametrized by some coordinate $\lambda$ of finite range. We can then perform the coordinate transformation $\boldsymbol{x} \rightarrow(\psi, \lambda)$, as illustrated in Fig. 9(a). One possible choice for $\lambda$ is the curve length along $R(\psi)$ from the external border of the design area. It is possible to show that if the permeability is uniform over the design area, the contour lines of $\psi$ are also field lines of $\boldsymbol{\nabla} H_{2}$. This implies that $H_{2}$ is monotonic along each $R(\psi)$ and is another possible choice for the parameter $\lambda$. We now write $\boldsymbol{H}_{2}(\boldsymbol{x})$ as $H_{2}(\boldsymbol{x}) \hat{\boldsymbol{e}}_{\psi}$, where $\hat{\boldsymbol{e}}_{\psi}=\cos (\psi) \hat{\boldsymbol{e}}_{x}+\sin (\psi) \hat{\boldsymbol{e}}_{y}$. Then the functional $\mathcal{S}$ can be written as

$\mathcal{S}=\sum_{n=1}^{N}\left\|\int d \psi \hat{\boldsymbol{e}}_{\psi} \int_{R(\psi)} d \lambda \phi_{n}(\boldsymbol{x}(\psi, \lambda)) H_{2}(\boldsymbol{x}(\psi, \lambda)) g(\psi, \lambda)\right\|$,

where $g(\psi, \lambda)$ is the absolute value of the determinant of the Jacobian of this transformation. Let us now define $h(\psi)$ as the total contribution to $\mathcal{S}$ from the set $R(\psi)$ :

$$
h(\psi)=\int_{R(\psi)} d \lambda H_{2}(\psi, \lambda) g(\psi, \lambda) .
$$

We also define $h_{n}(\psi)$ as the amount of $h(\psi)$ that is assigned to the $n$th segment:

$$
h_{n}(\psi)=\int_{R(\psi)} d \lambda \phi_{n}(\psi, \lambda) H_{2}(\psi, \lambda) g(\psi, \lambda) .
$$

Using the definition of the functions $\phi_{n}$, it can immediately be seen that $h_{n}(\psi) \geq 0$ for all $n$ and that the following relation holds for all $\psi$ :

$$
\sum_{n=1}^{N} h_{n}(\psi)=h(\psi)
$$

The functional $\mathcal{S}$ to be optimized can then be written as

$$
\mathcal{S}\left[\left\{\phi_{n}\right\}\right]=\sum_{n=1}^{N}\left\|\int d \psi h_{n}(\psi) \hat{\boldsymbol{e}}_{\psi}\right\| .
$$

The only dependence of $\mathcal{S}$ on $\phi_{n}$ is through the functions $h_{n}(\psi)$. Finding an optimal segmentation is then equivalent to finding a set of optimal functions $h_{n}(\psi)$. This fact makes the search space $\mathcal{X}$ equal to the space of all the $N$-dimensional vector functions defined over the interval of the real axis spanned by the values of $\psi$, subject to the conditions of non-negativity and the sum rule expressed in Eq. (A8). The sum between two elements $h^{(a)}$ and $h^{(b)}$ of $\mathcal{X}$ is defined in the usual way:

$$
\begin{aligned}
\text { if } h^{(c)} & =h^{(a)}+h^{(b)} \text { then } \\
h_{n}^{(c)}(\psi) & =h_{n}^{(a)}(\psi)+h_{n}^{(b)}(\psi), \quad \forall \psi, n .
\end{aligned}
$$

Similarly, multiplication of $h^{(a)}$ by the scalar $c$ is defined through

if $h^{(c)}=c h^{(a)} \quad$ then $h_{n}^{(c)}(\psi)=c h_{n}^{(a)}(\psi), \quad \forall \psi, n$.

These operations satisfy all the necessary properties that make $\mathcal{X}$ a vector space.

We can show that the functional $\mathcal{S}$ is convex with respect to $h_{n}(\psi)$. By definition, this means that if we consider two arbitrary vectors $h_{n}^{(a)}(\psi)$ and $h_{n}^{(b)}(\psi)$ and a scalar parameter $t \in[0,1]$, the following property is always obeyed:

$$
\begin{aligned}
& \mathcal{S}\left[t h^{(a)}+(1-t) h^{(b)}\right] \leq(t) \mathcal{S}\left[h^{(a)}\right]+(1-t) \mathcal{S}\left[h^{(b)}\right] \\
& \quad \leq \max \left(\mathcal{S}\left[h^{(a)}\right], \mathcal{S}\left[h^{(b)}\right]\right) .
\end{aligned}
$$


This property can be shown by considering each member of the summation over $n$ appearing in Eq. (A9) and applying the absolute homogeneity property of the norm and the triangle inequality.

We now restrict the search to a set $A \subset \mathcal{X}$ defined by the conditions of non-negativity and the sum rule of Eq. (A8):

$$
A=\{h_{n}(\psi): \underbrace{\sum_{n=1}^{N} h_{n}(\psi)=h(\psi)}_{\text {equality }}, \underbrace{h_{n}(\psi) \geq 0}_{\text {inequality }}, \forall \psi\} .
$$

We observe that the set $A$ is convex, since for any two points $h_{n}^{(a)}(\psi)$ and $h_{n}^{(b)}(\psi)$ belonging to the set and a value of $t \in[0,1]$, all the points on the line segment $t h^{(a)}+(1-t) h^{(b)}$ also belong to the set.

Let us then consider a particular solution $h_{n}^{(c)}$ for which there is at least one value of $\psi$ denoted $\psi^{\prime}$ for which $R\left(\psi^{\prime}\right)$ is subdivided between more than one segment. This assumption means that there exists an $n^{\prime}$ for which $h_{n^{\prime}}^{(c)}\left(\psi^{\prime}\right)$ is strictly between 0 and $h\left(\psi^{\prime}\right)$. We now define two additional points $h^{(a)}$ and $h^{(b)}$, which for $\psi \neq \psi^{\prime}$ are equal to $h_{n}^{(c)}$. For $\psi=\psi^{\prime}$, we set

$$
h_{n^{\prime}}^{(a)}\left(\psi^{\prime}\right)=0, \quad h_{n \neq n^{\prime}}^{(a)}\left(\psi^{\prime}\right)=h\left(\psi^{\prime}\right) \frac{h_{n}^{(c)}\left(\psi^{\prime}\right)}{\sum_{j \neq n^{\prime}} h_{j}^{(c)}\left(\psi^{\prime}\right)},
$$

and

$$
h_{n^{\prime}}^{(b)}\left(\psi^{\prime}\right)=h\left(\psi^{\prime}\right), \quad h_{n \neq n^{\prime}}^{(b)}\left(\psi^{\prime}\right)=0 .
$$

Evidently, the point $h^{(a)}$ corresponds to a segmentation identical to $h^{(c)}$ except that $R\left(\psi^{\prime}\right)$ is assigned entirely to the segment $n^{\prime}$. Similarly, $h^{(b)}$ has nothing of $R\left(\psi^{\prime}\right)$ assigned to $n^{\prime}$. The line segment $t h^{(a)}+(1-t) h^{(b)}$ for $t \in[0,1]$ is entirely inside the set $A$, but for $t<0$ or $t>1$, we are outside of $A$. The points $h^{(a)}$ and $h^{(b)}$ are, thus, on the boundary of $A$, and the original point $h^{(c)}$ is an interior point of the segment corresponding to $t=h_{n^{\prime}}^{(c)}\left(\psi^{\prime}\right) / h\left(\psi^{\prime}\right)$. Because of the convexity of $\mathcal{S}$, Eq. (A12) is obeyed for all the points on the line segment. Moreover, $t \mathcal{S}\left[h^{(a)}\right]+$ $(1-t) \mathcal{S}\left[h^{(b)}\right]$, which is the right-hand side of Eq. (A12), is always smaller than or equal to its value at one of the end points, $h^{(a)}$ or $h^{(b)}$. This argument is illustrated in Fig. 9(b) and shows that either $h^{(a)}$ or $h^{(b)}$ gives a value of $\mathcal{S}$ that is greater than or equal to $\mathcal{S}\left[h^{(c)}\right]$. Therefore, for any solution in which the set $R\left(\psi^{\prime}\right)$ is partially assigned to the segment $n^{\prime}$, there is a solution giving a greater or equal value of $\mathcal{S}$, for which $R\left(\psi^{\prime}\right)$ is either entirely assigned to the segment $n^{\prime}$ or not at all.

Thus, we show that assigning each set $R(\psi)$ entirely to a single segment gives a value of $\mathcal{S}$ which is equal to the maximum possible value achievable with $N$ segments.

We are now left with the problem of determining how to assign each $R(\psi)$ to one of the $N$ segments. However, until this point, we never enforced that the segments should be connected regions. This requirement implies that each segment corresponds to a single interval $\left[\psi_{n-1}, \psi_{n}\right]$ :

$$
h_{n}(\psi)=h(\psi), \quad \forall \psi \in\left[\psi_{n-1}, \psi_{n}\right] .
$$

The border between the segments $n$ and $n+1$ is, thus, given by the contour line $R\left(\psi_{n}\right)$, and the problem is reduced to the determination of the optimal splitting angles $\psi_{n}$ (see Appendix B).

\section{APPENDIX B: Optimal splitting angles}

In this section, we show how to select which of the contour lines of $\psi$ will lead to an optimal segmentation, given the total number of segments. Instead of $\arctan \left(H_{y} / H_{x}\right)$, we consider the accumulated angle $\psi$ informally introduced in Sec. III. In order to define the accumulated angle precisely, we consider a curve $\Gamma: t \in[0,1] \rightarrow \boldsymbol{x}(t)$, and we assume that $\boldsymbol{H}_{2}$ is rotating counterclockwise as we move along the curve. The purpose of $\Gamma$ is to create a one-to-one correspondence between the contour lines $R\left(\psi_{n}\right)$ and the continuous parameter $t \in[0,1]$. The accumulated angle is defined for each $t$ as the angle $\psi$ which is obtained by shifting $\arctan \left(H_{y} / H_{x}\right)$ by a number of complete revolutions, which is determined by requiring monotonicity and continuity. The monotonicity property means that $(d / d t) \psi[\boldsymbol{x}(t)]>0, \forall t \in[0,1]$. The continuity property means that if $t_{a} \rightarrow t_{b}$, then $\psi\left(t_{a}\right) \rightarrow \psi\left(t_{b}\right)$. If the curve $\Gamma$ is closed, we also have the following continuity property across the starting point: if $t_{a} \rightarrow 0^{+}$and $t_{b} \rightarrow 1^{-}$, then $\psi\left(t_{b}\right)-\psi\left(t_{a}\right) \rightarrow n 2 \pi$, where $n$ is the number of revolutions performed by $\boldsymbol{H}_{2}$ from the first point $t=0$ to the last point $t=1$. The curve $\Gamma$ can be constructed by starting from an arbitrary point and proceeding in a way such that each $R(\psi)$ is intersected once and only once. However, as long as $\psi$ is monotonic, the result is the same for all the choices of $\Gamma$.

Since we assume that the union of all the $\psi$ contour lines passing by the points of the curve $\Gamma$ is coincident with the whole design area $R_{m}$, the definition of the accumulated angle $\psi$ can be extended to any other point of $R_{m}$ that is not on $\Gamma$.

We now define the region $\mathcal{R}_{\left[\psi_{0}, \psi_{1}\right]}$ as the set of points $\boldsymbol{x}$ in the magnet design area in which the orientation angle $\psi$ of the virtual field $\boldsymbol{H}_{2}$ is in the interval $\left[\psi_{0}, \psi_{1}\right]$,

$$
\mathcal{R}_{\left[\psi_{0}, \psi_{1}\right]}=\left\{\boldsymbol{x}: \psi\left[\boldsymbol{H}_{2}(\boldsymbol{x})\right] \in\left[\psi_{0}, \psi_{1}\right]\right\} \cap R_{m} .
$$




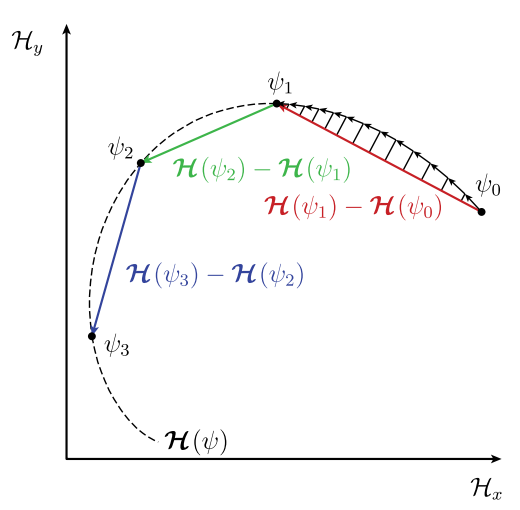

(a)

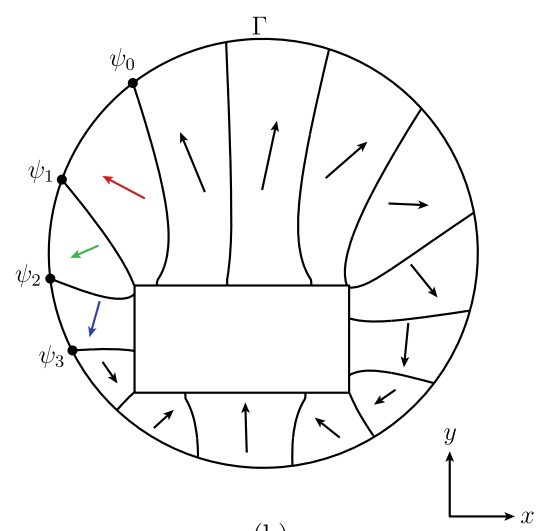

(b)
FIG. 10. In the continuous case, the optimal remanence is normalized and aligned at any point with the virtual field. This implies that the value of $\mathcal{S}$ is given by the length of the curve $\mathcal{H}(\psi)$. In the segmented case, the contribution from a point belonging to a certain segment is proportional to the scalar product between the virtual field and the optimal remanence for that segment. This implies that the direction of the vector $\mathcal{H}\left(\psi_{1}\right)-\mathcal{H}\left(\psi_{0}\right)$ is also the optimal direction of the remanence, and its length is equal to the contribution from that segment to the total value of $\mathcal{S}$. (a) The curve $\mathcal{H}(\psi)$ and its segmentation. (b) The corresponding segmented geometry.
The regions so defined satisfy $\mathcal{R}_{\left[\psi_{0}, \psi_{2}\right]}=\mathcal{R}_{\left[\psi_{0}, \psi_{1}\right]} \cup \mathcal{R}_{\left[\psi_{1}, \psi_{2}\right]}$ for all $\psi_{1} \in\left[\psi_{0}, \psi_{2}\right]$.

The integrated magnetic field vector associated with this region is

$$
\mathcal{H}_{\left[\psi_{0}, \psi_{1}\right]}=\int_{\mathcal{R}_{\left[\psi_{0}, \psi_{1}\right]}} d^{2} x \boldsymbol{H}_{2}(\boldsymbol{x})=\int_{\psi_{0}}^{\psi_{1}} d \psi h(\psi) \hat{\boldsymbol{e}}_{\psi}
$$

with $h(\psi)$ defined in Eq. (A6). The symbol $\mathcal{H}$ denotes the integral of a field over a region of space. These vectors satisfy the property $\mathcal{H}_{\left[\psi_{0}, \psi_{2}\right]}=\mathcal{H}_{\left[\psi_{0}, \psi_{1}\right]}+\mathcal{H}_{\left[\psi_{1}, \psi_{2}\right]}$ for all $\psi_{1} \in\left[\psi_{0}, \psi_{2}\right]$.

Once a starting point $\psi_{0}=\psi[\boldsymbol{x}(t=0)]$ is fixed, it is possible to parametrize the vectors associated with the different regions by just using the value $\psi$ of the ending point; the following notation is used: $\mathcal{H}(\psi)=\mathcal{H}_{\left[\psi_{0}, \psi\right]}$. The velocity vector associated with this parametrized curve is denoted by $\boldsymbol{h}(\psi)=(d / d \psi) \mathcal{H}(\psi)=h(\psi) \hat{\boldsymbol{e}}_{\psi}$.

An example geometry and the corresponding curve $\mathcal{H}(\psi)$ are shown in Figs. 10(b) and 10(a), respectively.

In the continuous case, the contribution to $\mathcal{S}$ from the interval $\left[\psi_{0}, \psi_{1}\right]$ is equal to the length of the corresponding arc of curve:

$$
\begin{aligned}
\mathcal{S}_{\text {continuous }} & =\int_{\mathcal{R}_{\left[\psi_{0}, \psi_{1}\right]}} d^{2} x\left\|\boldsymbol{H}_{2}(\boldsymbol{x})\right\| \\
& =\int_{\psi_{0}}^{\psi_{1}} d \psi\|\boldsymbol{h}(\psi)\| \\
& =\int_{\psi_{0}}^{\psi_{1}} d \psi h(\psi) .
\end{aligned}
$$

In the segmented case, the contribution to $\mathcal{S}$ is the length of the segment between the curve end points

$$
\begin{aligned}
\mathcal{S}_{\text {segmented }} & =\left\|\int_{\mathcal{R}_{\left[\psi 0, \psi_{1}\right]}} d^{2} x \boldsymbol{H}_{2}(\boldsymbol{x})\right\| \\
& =\left\|\int_{\psi_{0}}^{\psi_{1}} d \psi \boldsymbol{h}(\psi)\right\|=\left\|\mathcal{H}\left(\psi_{1}\right)-\mathcal{H}\left(\psi_{0}\right)\right\| .
\end{aligned}
$$

When considering more than one segment, the value of $\mathcal{S}_{\text {segmented }}$ is given by the length of the polygonal line inscribed in $\mathcal{H}(\psi)$.

The problem of optimal segmentation is reduced to the problem of piecewise linear approximation of plane curves by perimeter optimization.

The globally optimal solution for this problem can always be found by employing a dynamic programming approach. This class of algorithms makes use of the optimal substructure exhibited by the problem in order to reduce its computational complexity. An algorithm for the curve approximation problem can be found in Ref. [24].

If the curve $\Gamma$ is closed, it is possible to consider the dependence of $\mathcal{S}$ on the starting point $\psi_{0}$ to determine the optimal starting point. A necessary but not sufficient condition for this is that the design area encloses the air gap completely. Once the curve $\mathcal{H}(\psi)$ is computed, this is not a computationally intensive procedure and can be performed as the last step of the optimization.

It is worthwhile to mention that the procedure described in this section can be generalized to different continuously parametrized segmentations in order to meet other design requirements. In this case, the final configuration is only optimal with respect the considered search space. Let us consider the simple example of a rectangular design area $R_{m}$ and the family of rectangular subsets:

$$
\mathcal{R}_{\left[x_{0}, x_{1}\right]}=\left\{\boldsymbol{x}: x \in\left[x_{0}, x_{1}\right]\right\} \cap R_{m} .
$$

The curve $\mathcal{H}(x)$ can be constructed from this parametrized set of rectangles as in Eq. (B2). The optimal segmentation of this curve with a given number of segments provides the set of values of $x$ corresponding to the optimal splitting lines among the ones allowed by Eq. (B5). Any family of regions defined by a single parameter and obeying the same properties as described above can be considered with the purpose of constraining the shapes of the resulting segments as desired, by relaxing the condition of having an optimal shape of the border between two adjacent segments. 
[1] O. Gutfleisch, M. A. Willard, E. Brück, C. H. Chen, S. G. Sankar, and J. P. Liu, Magnetic materials and devices for the 21st century: Stronger, lighter, and more energy efficient, Adv. Mater. 23, 821 (2011).

[2] J. M. D. Coey, Permanent magnet applications, J. Magn. Magn. Mater. 248, 441 (2002).

[3] K. Yamazaki and Y. Kanou, Shape optimization of rotating machines using time-stepping adaptive finite element method, IEEE Trans. Magn. 46, 3113 (2010).

[4] L. Yan, I. M. Chen, C. K. Lim, G. Yang, W. Lin, and K. M. Lee, Design and analysis of a permanent magnet spherical actuator, IEEE/ASME Trans. Mechatron. 13, 239 (2008).

[5] J. Schönke, Smooth Teeth: Why Multipoles Are Perfect Gears, Phys. Rev. Applied 4, 064007 (2015).

[6] H. Zijlstra, Permanent magnet systems for NMR tomography, Philips J. Res. 40, 259 (1985).

[7] G. Moresi and R. Magin, Miniature permanent magnet for table-top NMR, Concepts Magn. Reson. 19B, 35 (2003).

[8] S. Becker, M. Bussmann, S. Raith, M. Fuchs, R. Weingartner, P. Kunz, W. Lauth, U. Schramm, M. El Ghazaly, F. Grüner, H. Backe, and D. Habs, Characterization and Tuning of Ultrahigh Gradient Permanent Magnet Quadrupoles, Phys. Rev. ST Accel. Beams 12, 102801 (2009).

[9] B. Biswas, A magnetic quadrupole from rectangular permanent magnets, Nucl. Instrum. Methods Phys. Res. 605, 233 (2009).

[10] B. Diviacco, Performance optimization of pure permanent magnet undulators, Proc. IEEE Part. Accel. Conf. 2, 1590 (1993).

[11] R. Bjørk, C. R. H. Bahl, A. Smith, and N. Pryds, Improving magnet designs with high and low field regions, IEEE Trans. Magn. 47, 1687 (2011).

[12] S. Cheng and D. P. Arnold, Optimization of permanent magnet assemblies using genetic algorithms, IEEE Trans. Magn. 47, 4104 (2011).

[13] M. Markovic and Y. Perriard, Optimization design of a segmented Halbach permanent-magnet motor using an analytical model, IEEE Trans. Magn. 45, 2955 (2009).

[14] R. Ravaud, G. Lemarquand, and V. Lemarquand, Magnetic field created by a uniformly magnetized tile permanent magnet, Prog. Electromagn. Res. B 24, 17 (2010).
[15] M. G. Abele, H. Rusinek, F. Bertora, and A. Trequattrini, Compensation of field distortion with ferromagnetic materials and permanent magnets, J. Appl. Phys. 75, 6990 (1994).

[16] M. G. Abele, W. Tsui, and H. Rusinek, Methodology of pole piece design in permanent magnets, J. Appl. Phys. 99, 08 D903 (2006).

[17] W. F. Brown, Magnetostatic Principles in Ferromagnetism (North-Holland Publishing Company, Amsterdam, 1962).

[18] N. I. Klevets, Synthesis of magnetic systems producing field with maximal scalar characteristics, J. Magn. Magn. Mater. 285, 401 (2005).

[19] N. I. Klevets, Optimal design of magnetic systems, J. Magn. Magn. Mater. 306, 281 (2006).

[20] A. R. Insinga, C. R. H. Bahl, R. Bjørk, and A. Smith, Performance of Halbach magnet arrays with finite coercivity, J. Magn. Magn. Mater. 407, 369 (2016).

[21] K. Halbach, Design of permanent multipole magnets with oriented rare earth cobalt material, Nucl. Instrum. Methods 169, 1 (1980).

[22] C. K. Chandrana, J. A. Neal, D. Platts, B. Morgan, and P. Nath, Automatic alignment of multiple magnets into Halbach cylinders, J. Magn. Magn. Mater. 381, 396 (2015).

[23] J. H. Jensen and M. G. Abele, Maximally efficient permanent magnet structures, J. Appl. Phys. 79, 1157 (1996).

[24] Y. Sato, Piecewise linear approximation of planar curves by perimeter optimization, Pattern Recogn. 25, 1535 (1992).

[25] R. Bjørk, A. Smith, and C. R. H. Bahl, Analysis of the magnetic field, force, and torque for two-dimensional Halbach cylinders, J. Magn. Magn. Mater. 322, 133 (2010).

[26] M. G. Abele, Linear theory of yokeless permanent magnets, J. Magn. Magn. Mater. 83, 276 (1990).

[27] M. G. Abele and H. Rusinek, Optimum design of yokeless permanent magnets, J. Appl. Phys. 67, 4644 (1990).

[28] M. G. Abele, Structures of Permanent Magnets (John Wiley and Sons, New York, 1993).

[29] R. Bjørk, C. R. H. Bahl, A. Smith, and N. Pryds, Optimization and improvement of Halbach cylinder design, J. Appl. Phys. 104, 013910 (2008). 